\title{
High Throughput and Thermal Aware Routing Protocol (HTTRP) for Wireless Body Area Networks
}

\author{
Samir Bouldjadj*, Zibouda Aliouat \\ Computer Science Department, Laboratory LRSD, Ferhat Abbas University - Setif 1, Setif 19000, Algeria
}

Corresponding Author Email: samir.bouldjadj@univ-setif.dz

https://doi.org/10.18280/isi.250106

Received: 9 October 2019

Accepted: 26 December 2019

\section{Keywords:}

energy-aware, routing protocols, thermalaware, throughput, wireless body area networks

\begin{abstract}
One of the major applications of sensor networks in the near future will be in the area of biomedical research. Wireless Body Area Networks (WBANs) are composed of implanted biosensors for health monitoring and diagnostic purposes. The communication between these sensors is made in a wireless way at the base of the radio waves. The sensors' activity produces heat causing a temperature rise. The high-temperature rise of the sensors for a prolonged period might damage the surrounding tissues. Various routing protocols have been proposed in the literature to remedy this problem. These protocols tried to perform routing based on the SHR algorithm while avoiding hot-spots nodes. However, the energy of sensor nodes located in this shorter path is quickly exhausted and by the way, the whole network lifetime is influenced. In this work, we propose HTTRP, a new routing protocol for WBANs introducing a new route selection mechanism that aims to reduce the overheating of sensors and balance their energy consumption. This mechanism is based on a function that considers the residual energy of sensor nodes and their temperature when choosing the next relay node. The carried out simulation results show that our HTTRP protocol has better performance in terms of network lifetime, charge balancing, temperature rise, and throughput compared to a representative of TARP that is TARA protocol.
\end{abstract}

\section{INTRODUCTION}

The rapid development of sensors, low-power integrated circuits and wireless communications has led to the emergence of a new generation of wireless sensor networks. These wireless sensor networks are used for the control of various activities, entertainment or health $[1,2]$.

The Wireless Body Area Network (WBAN) [3-5] is a particular type of wireless sensor network (WSN). WBAN is used in several fields [6] notably in the health field. It allows through miniature biomedical sensors ported or implanted on patients to collect vital information like heartbeat, blood pressure and body temperature. This information is sent to the remote care center to take necessary actions immediately, all this at a very low cost. WBAN can contribute to the improvement of patient care and early detection of health impairments [7]. To ensure this functionality, the WBAN nodes need to communicate with each other, this task is ensured by the routing protocol.

Recent advances in WBANs have led to the development of many routing protocols specifically designed for this type of network where QoS, mobility, security, and sensor nodes' temperature are considered as routing metrics [6]. One of the major challenges that routing protocols designed for WBANs must address is the reduction of overheating of implanted sensors. The overheating is due to radiation from the sensors' antennas and the energy consumption at their circuits (dissipation of electrical energy in the form of heat). The generated heat is likely to be fatal to the body carrying the WBAN $[6,8]$. Researchers have proposed many protocols that consider sensor nodes' temperature as a routing parameter.
These protocols are called Thermal-Aware Routing Protocols it $[6,9,10]$. Most of these protocols aim to reduce the overheating of sensor nodes by avoiding hot spots when selecting routing paths. These protocols use in most cases the shortest paths to route the data, however, the shortest path is not always optimal in terms of energy-saving because nodes located in the shortest path suffer from overload and exhaust quickly, which may affect the functioning and the existence of the whole network.

In this work, our purpose is to design HTTRP, a routing protocol that uses another way (criterion) for routing paths selection. HTTRP tries to extend the network lifetime by balancing the energy consumption of sensor nodes while avoiding the increase of nodes' temperature. For this purpose, HTTRP uses a combination of two metrics that are nodes' energy and temperature as a routing metric when selecting paths.

The rest of the paper is organized as follows: in Section 2, related work is provided. Section 3 explains the background and motivation. Section 4 contains a brief explanation of our proposed protocol. Section 5 is provided with the performance evaluation and comparison of the proposed protocol and TARA protocol, finally, section 6 gives the conclusion.

\section{RELATED WORK}

Radio signals used by wireless communication generate magnetic and electric fields. Exposure to these electromagnetic fields causes the absorption of radiation by the human tissue [11]. This can affect heat-sensitive organs in 
reducing blood flow, the growth of certain tissues, enzymatic reactions ... etc. The amount of energy radiation absorbed by human tissue is referenced as the Specific Absorption Rate (SAR) [12].

To avoid node's overheating problem, Temperature-Aware Routing Protocols $[13,14]$ are proposed, those are routing protocols that take into account node temperature as metric in selecting routing path. In what follows we will focus on the routing protocols of the class Temperature-aware routing protocols, the most well-known existing protocols of this class include TARA [15], LTR, ALTR [16], LTRT [17], HPR [18], RAIN [19], TSHR [20], TMQoS [9], M2E2 [21], TTRP [22] and ATAR [23].

The first protocol of this class is The thermal-aware routing algorithm (TARA) [15], this protocol identifies nodes with temperature superior of a threshold value as hotspots, those nodes are avoided during routing phase which consists of sending packets from nodes to sink, and if the neighbor nodes are host-spots, the packet is tamponed until node temperature decrease. Before that, in the initialization phase, each node observes the activities of neighboring nodes to measure the changes in their temperatures, and that by counting the received/ transmitted packets, evaluating their communication radiation. Although this strategy reduces the problem of overheating, it causes a high routing delay which influences network lifetime as the packet goes through the network for a considerable time.

Least temperature routing (LTR) [16], is very similar to TARA, especially in the initialization step. Hence packet counts the number of hops passed. If this number exceeds the predetermined value denoted MAXHOPS, the current packet is discarded and is rerouted using an alternate route. The no redundancy of routes is guaranteed by maintaining tracks of all passing packets in each node, so each node tries to transmit the packet to the coldest not visited neighbor node.

Adaptive Least Temperature Routing (ALTR) [16] is formulated from LTR. The main amelioration is if the value of hops number exceeds the predetermined value MAXHOPS, the packet is routed using the SHR algorithm, if neighboring nodes are hotspots, the current node will wait a unit of time for nodes to cool down.

Least Total Route Temperature (LTRT) [17] is another TARP, which is a combination of the LTR and SHR protocol. This protocol is designed to find paths with a minimum total temperature as well as to solve the problem of redundant hops. First, the estimated temperatures of each node are assigned as weights to the route between two sensor nodes, and then Dijikstra's shortest path algorithm is applied on a labeled graph of weight obtained to find routes that have the least temperature from the source to the destination. The route is updated periodically to avoid the excessive rise of the node's temperature.

Hotspot Preventing Routing (HPR) [18] is an improvement of LTR and ALTR. With LTR and ALTR, the reduction of the average temperature of the network does not necessarily prevent any node from having a very high temperature. However, HPR not only prevents the formation of hotspots in the network but it prevents also packets to take suboptimal paths and thus reduces the average time, it is suitable for delaysensitive applications. This protocol achieves the two goals of hotspot prevention by using a threshold value and delay reduction using the SHR algorithm.

The routing algorithm for networks of homogeneous and ID-less biomedical sensor nodes (RAIN) [19] is published in
2008. The ID-less term used in this protocol means that nodes use temporary identifiers. IDs are randomly generated during the configuration phase, the 'zero' ID is reserved for the sink node. All nodes, including the sink, broadcast their identifiers via "Hello" packets. The multi-hop technique is used for routing packets, where each packet has a unique packet identifier ID [N, T, R] where ' $\mathrm{N}$ ' is the node ID, ' $\mathrm{T}$ ' is the packet's time generation at the source node and ' $R$ ' is a random number. Each node saves a list of identifiers of visited packets and this to avoid duplicate packet transmission, so node drops packet if it's ID already exists in this list. A packet is also dropped if its hop-count exceeds threshold "HOP_THRESH" and this to stop the packet's infinite routing loops. If the destination node is among the neighboring nodes, the packet is directly transmitted; otherwise, it is transmitted to the neighbor node using a probability that is inversely proportional to its temperature. When receiving a packet by the sink, he broadcasts an update message containing the identifier of the packet to all its neighboring nodes and this to stop sending multiple copies of the same packet.

Thermal-Aware Shortest Hop Routing (TSHR) [20] is an improved version of HPR. TSHR has been designed for applications that require high-priority data, dropped packets for any reason are instantly retransmitted. Nodes route packets to the destination using the shortest path. To control the temperature of nodes, TSHR uses two thresholds: Ts and Tdn, Ts: is a fixed static threshold that the temperature of nodes must not exceed. Tdn: A dynamic threshold defined for a node as a function of the temperature of the neighboring nodes and its temperature. A node is declared as a hotspot if its temperature is higher than the Tdn which is calculated as follows:

$$
\text { Tdn=tempn }+0.25 \sqrt{\text { tempn }}+0.25 \sqrt{\text { avgn }}
$$

where, tempn is the temperature of the node and avgn is the average temperature of its neighbors.

Thermal-Aware Multiconstrained Intrabody QoS Routing (TMQoS) [9], Proposed in 2014 is the first protocol integrating the support of quality of service, it considers several QoS metrics such as delay and reliability as well as temperature and hops count as routing metrics. TMQoS meets the desired QoS requirements for different types of data. Whatever the type of data, TMQoS chooses paths by avoiding hotspots, which allows it to keep the temperature rise to an acceptable level. Nodes exchange a beacon packet for the construction and maintenance of the routing table that contains routes to sink with the minimum hop count. It has several modules; The delay estimator, the reliability estimator and the temperature estimator which measures the node temperature. The routing table constructor module implements a hotspot avoidance mechanism that keeps the packet away from the hotspot area.

Trust and Thermal Aware Routing Protocol (TTRP) [22], proposed in 2017. This protocol ensures trustiness and avoids hotspot formation in the network. It uses specified nodes dotted with high energy served only as data-relay for other nodes. TTRP protocol implements three phases; trust estimation phases, routing discovery phases and routing maintenance phases. The phase named trust estimation is responsible for estimating the trustiness of relay nodes. The route discovery phase has the task to find out in the network a route ensuring trust and hotspot free to use for data transmission. The Last phase is the route maintenance phase, which deals with link failure, if a node becomes hotspot during 
communication or if the neighboring node becomes untrusted, its re-initiates the route discovery phase.

Adaptive Thermal-Aware Routing Protocol (ATAR) [23], proposed in 2019. This protocol tries to balance the load among sensor nodes in the context of temperature rise. It implements two phases; initialization phase and data transmission phase. The initialization phase consists of classifying nodes in ring levels that depict their distance to the sink in terms of hop-count. In data transmission phase, the node selects relay node with the least rank value (node's temperature) as a next hop, and broadcasts the data packet containing its ring level, its temperature and relay node ID, when the specified relay node receives the data packet, it rebroadcast the data packet. However, Nodes with greater ring level keep ID and temperature of the source node and discard the packet, others nodes instantly discard the packet, this process continues until the packet is received by the sink. Like TTRP, ATAR uses specified nodes having high energy that serves only as data-relay for other nodes.

\section{BACKGROUND AND MOTIVATION}

Like TARA [15] protocol, others TAR protocols (thermal aware routing) LTR [16], ALTR [16], LTRT [17], HPR [18], RAIN [19], TSHR [20], TMQoS [9] did not consider the problem of energy consumption. They implicitly try to address the problem by (a) reducing packet duplication (RAIN [19]). (b) choosing the shortest routing paths (like HPR [18] and TSHR [20]). However, the shortest path is not always optimal in terms of energy consumption because nodes located in the shortest path suffer from overload and exhaust quickly, which may affect the functioning and existence of the network (Figure 1). (c) Another solution adopted by some recent protocols like TTRP and ATAR stipulates the existence of relay nodes whose only task is to receiving and forwarding packets while sensor nodes only collect and transmit their collected data. This technique can prolong the network lifetime by reducing the energy consumption of sensor nodes. At the same time, the network lifetime is highly affected by these relay nodes, also adding extra equipment on the human body may affect people's and disturb their comfort. For all these reasons, nodes' energy must be considered as a crucial metric when selecting routing paths in the thermal aware routing protocols.

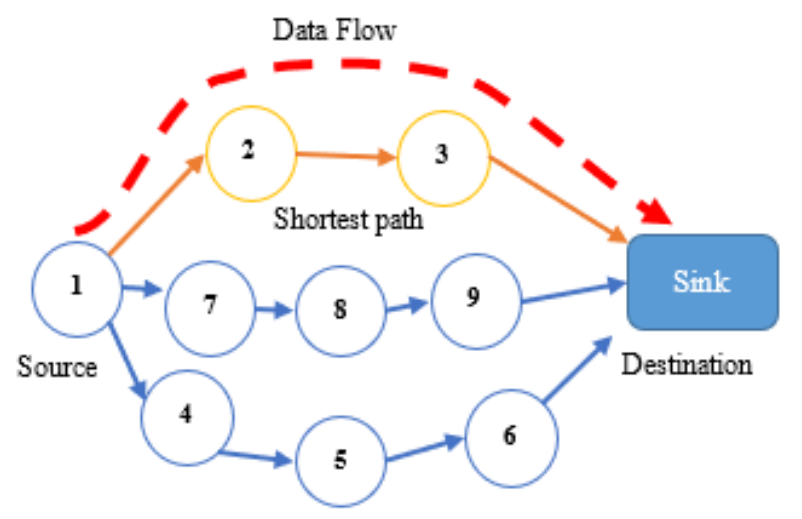

Figure 1. Nodes in the shortest path die quickly

With TARA, RAIN, TSHR, ... and others thermal aware routing protocols, data is sent via the shortest path, so nodes forming this path are going to run out quickly.

\section{PROPOSED PROTOCOL}

\subsection{Presentation of HTTRP}

We propose HTTRP (High Throughput and Thermal aware Routing Protocol) which is a thermal and Energy-Aware Routing Protocol. It is an algorithm that selects the routing path on the base of the node's temperature and energy. In HTTRP, the routing path is selected not only according to the temperature (like others TAR protocols) but also according to the energy level of the sensor nodes implanted in the body. Thus, it aims to reduce the overheating of sensor nodes that can cause tissue damage and to extend the network's lifetime by good energy management.

\subsection{System model and assumptions}

In our model, we assume that:

- The locations of biosensors are predefined since they are physically located rather than randomly as in other applications of sensor networks and the location of neighboring is known to each other after the initialization phase.

- Sensors are implanted in the subcutaneous tissue and periodically generate packets that must be sent to the sink node, the latter aggregates the data and transmits them to a base station, which is located outside the body. The sink has enough continuous energy supply.

- Each node has an omnidirectional antenna and no sensor node is disconnected from the network. Nodes exchange a fixed-sized packet and each node transmits data at its time slot.

- The topology is mesh, each sensor node has static transmission power and transmission range. Emergency data is sent directly to the sink. However, for normal data, the nodes must make the multi-hop to route the data towards the sink.

\subsection{Description of HTTRP protocol}

In our protocol, data transmission is based on local information about the temperature and energy of sensor nodes. A node whose temperature exceeds a threshold (predefined value) is marked by its neighbors as a hotspot. In the following subsection, we describe the principles steps of our protocol.

\subsubsection{Initialization phase}

In this phase, the sink node initiates a HELLO message containing information about neighbors in the form of hop count from sink, temperature and energy level as shown in Figure 2 and broadcast it. On receiving the HELLO message, the node keeps the containing data in its routing table, updates the HELLO message and rebroadcasts it. Thus, at the end of this phase, each node can establish its list of neighbors with their respective hope-count to sink, temperature and energy level.

\begin{tabular}{|l|l|l|}
\hline Neighbor_ID & Residual Energy & Temperature \\
\hline
\end{tabular}

Figure 2. Hello message format

\subsubsection{Routing phase}

In this phase, captured data by different nodes must be sent 
to the sink. In our approach, we consider two types of data, the emergency data and the normal data. For the emergency data and because of its urgent nature, the nodes send it directly to the sink using single-hop communication. However, the transmission of normal data is based on two parameters: node's temperature, which must be minimal, and residual energy, which must be maximal. In order to ensure the establishment of a path that reduces the overheating of sensor nodes and balances the energy expenditure by nodes during data transmission, we have combined two criteria for selecting the next relay node by defining an objective function $\boldsymbol{F i}(\boldsymbol{T}, \boldsymbol{E})$ which represents the new criterion for the selection of the next relay node. Thus, a node selects from its neighborhood table the non-hotspot neighbor having the maximum value of the $F i$ function which is defined as follows:

$$
F i(T, E)=\frac{E_{i}}{T_{i}}
$$

where, $E i$ is the residual energy of the neighbor node i and $T i$ is its temperature.

\begin{tabular}{|l|l|l|l|}
\hline Sender_ID & Residual Energy & Temperature & Dest_ID \\
\hline
\end{tabular}

Figure 3. Acknowledgment message format

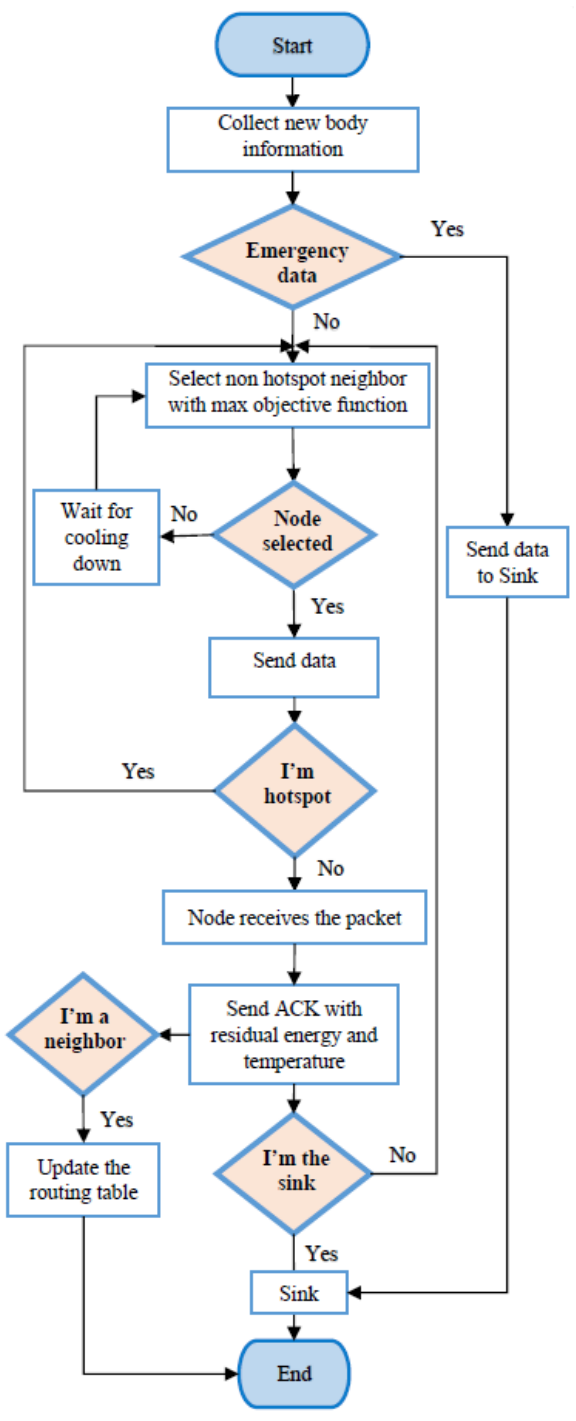

Figure 4. Flow diagram of HTTRP

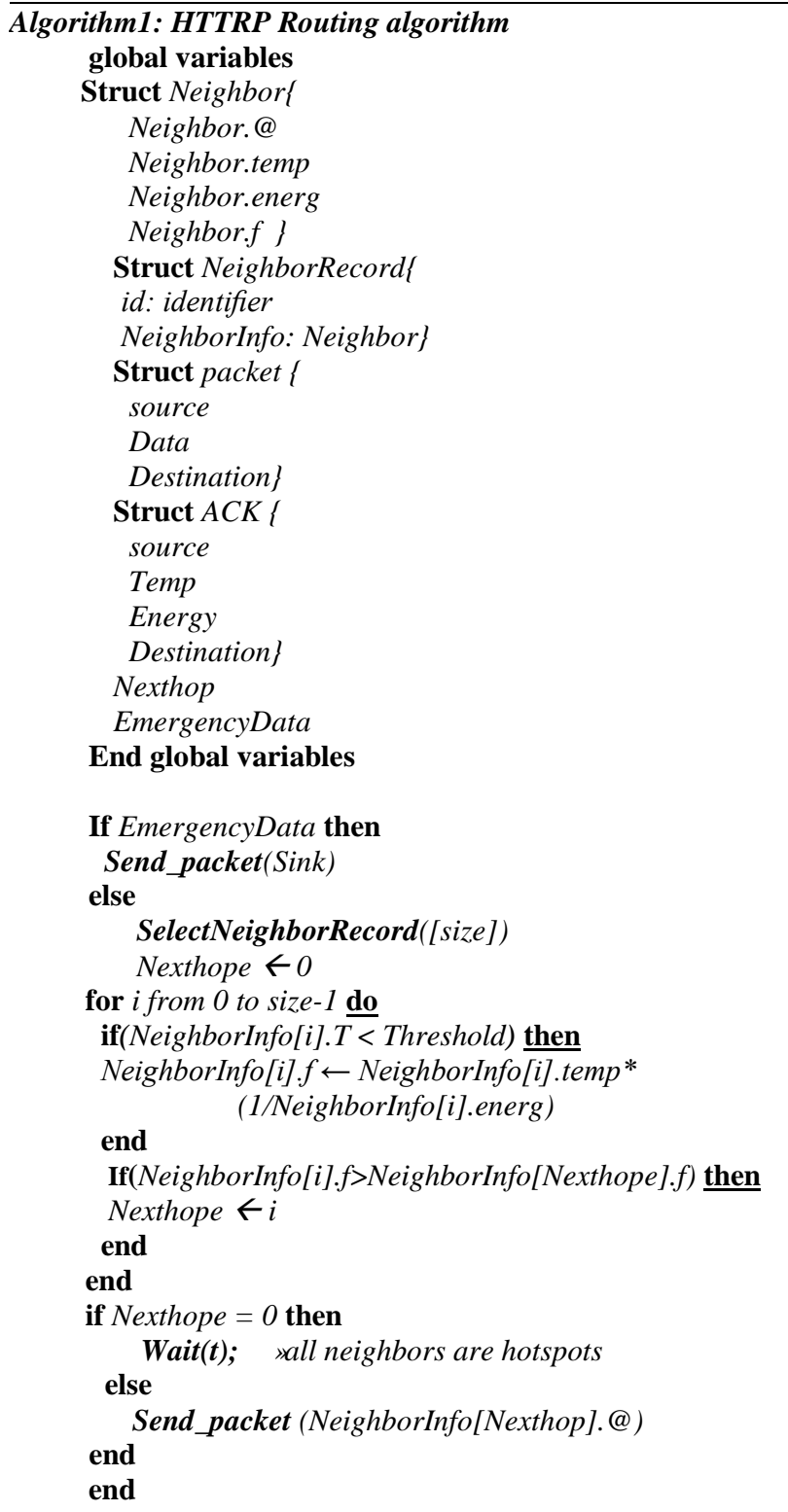

Receiving a Data Packet

if node. $T>$ threshold then
send $($ ACK)
else
discardPacket();
endif

In order to save node's energy, we adopt the below strategies: A node sends his captured data only if it is different from the previously sent data; in other words, nodes spend their energy only for utile data. The acknowledgment packet is used efficiently, to explain the principle, let's consider $N s$ a sender node a $\mathrm{Nr}$ the recipient node. In the normal case, when $\mathrm{Nr}$ receives a packet from $\mathrm{Ns}, \mathrm{Nr}$ sends an acknowledgement packet in a short time Tack, containing $\mathrm{Nr}$ residual energy and $\mathrm{Nr}$ temperature (Figure 3). On receiving the acknowledgment packet, $N s$ updates the corresponding information in its routing table, also all nodes whose receive this acknowledgment packet update their routing table. If $N r$ is a hotspot (its temperature exceeds a predefined threshold value), $\mathrm{Nr}$ will not acknowledge $N s$ (this to conserve node temperature and 
accelerate the cooling down operation). After the expiration of Tack, $\mathrm{Ns}$ understands that $\mathrm{Nr}$ is a hotspot, and by the way, it marks it as a hotspot, then it tries to send the packet to another available hop. If all neighbors are hotspots, in this case, $N s$ must wait them for cooling down for a fixed time period, if a hotspot node does not do any activity for this time period, its temperature will drop and again it can be solicited for the routing operation.

The principle of our HTTRP protocol is illustrated in Figure 4 by a Flow diagram.

\subsection{Implementation and simulation}

\subsubsection{Energy consumption analysis}

In WSN, the energy consumed by a sensor node is essentially due to the following operations: data capturing, data processing and data communication. Several models of energy consumption have been proposed to study and evaluate the different types of energy dissipation in these networks [17, $24,25]$. Due to high-energy consummation by the data communication, most of energy consumption models focus more on the evaluation of communication energy. The amount of energy consumed depends on the mode of communication used: single-hop or multi-hop.

Heinzelman et al. [24] proposed an energy consumption model that only considers transmission and reception energies. Thus, according to this model, to transmit a message of length $k$ bits between a transmitter and a receiver at a distance $d$ (in meters); the radio module of the transmitter spends the energy quantity (in Joules) given by the following Eq. (2):

$$
E_{T X}(k, d)=E_{T X-e l e c}(k)+E_{T X-a m p}(k, d),
$$

To receive this message, the radio module of the receiver expends the energy quantity (in Joules) given by the following
Eq. (3):

$$
E_{R x}(k)=E_{R x-e l e c}(k),
$$

In these two equations, $E_{T x-e l e c}$ and $E_{R x-e l e c}$ respectively represent the electronic energy of the transmitter and that of the receiver.

$$
\begin{gathered}
E_{T X \text {-elec }}=E_{R X-e l e c}=E_{\text {elec }}=50 \mathrm{~nJ} / \mathrm{bit}, \\
\varepsilon_{\text {amp }}=100 \mathrm{pJ} / \mathrm{bit} / \mathrm{m}^{2},
\end{gathered}
$$

$\varepsilon_{\text {amp }}$ is the energy used by the transmit amplifier. It is the most critical component in transmitter subsystem, that is, a major source of energy drainage. More details can be found in the study [26].

According to Eqns. (2), (3), (4) and (5), the energy quantities expended for transmission $\left(T_{X}\right)$ and reception $\left(R_{X}\right)$ of a message of length $k$ bits at a distance $d$ can be written respectively:

$$
\begin{gathered}
E_{T X}(k, d)=E_{\text {elec }}{ }^{*} k+\varepsilon_{\text {amp }}{ }^{*} k^{*} d^{2} \\
E_{R x}(k)=E_{\text {elec }}{ }^{*} k
\end{gathered}
$$

\subsubsection{Simulation model}

In purpose to evaluate the performance of our proposed protocol HTTRP, we used MATLAB simulations based on the assumptions made in the system model and assumptions section and using the parameters described in Table 2 in which it is compared with TARA protocol in terms of network lifetime, charge balancing, throughput and the number of generated hotspots. The energy consumption is calculated using the model described in the previous section 4.4.1.

Table 1. Biomedical sensors used in the simulation model

\begin{tabular}{ccl}
\hline Biomedical sensor & Signification & \multicolumn{1}{c}{ Function } \\
\hline EEG & Electro Encephalo-Gram & Records electroencephalographic signals from the brain \\
\hline ECG & Electro Cardio Gram & Records an electrical signal produced by the heart \\
\hline TENS & Blood Pressure & Measures of the force that blood exerts against the walls of blood vessels \\
\hline EMG & Electro Myo Gram & Records electromyography signals from different muscles in the body \\
\hline MS & Motion Sensor & Detects movement alerting medical staff that the patient is moving. \\
\hline SINK & The sink node & The base station \\
\hline AK & Artificial Knee & Artificial knee \\
\hline Glu & Glucose & Measures the blood glucose concentration \\
\hline BO & Blood oxygen & Used for measurements of arterial blood gas \\
\hline InInj & Insulin Injector & Used for Insulin Injection \\
\hline Pos & Positioning & Used for remote surgical tool positioning and patient bed positioning \\
\hline LA & Lactic Acid & Used for lactic acid sensing \\
\hline
\end{tabular}

Table 2. Simulation parameters

\begin{tabular}{cccc}
\hline Parameter & Definition & Initial Value & Unit of measure \\
\hline $\mathrm{x}, \mathrm{y}$ & Coordinates of a node & Predefined Position & Meter \\
\hline E0 & Initial energy & {$[0,0.1]$} & Joule \\
\hline ETx & Transmission energy & 50 & Nano Joule/bit \\
\hline ERx & Reception energy & 50 & Nano Joule/bit \\
\hline Eelec & Electronic energy & 50 & Nano Joule/bit \\
\hline Amp & Amplification factor & 100 & $\mathrm{pJ} / \mathrm{bit} / \mathrm{m}^{2}$ \\
\hline P_size & Packet size & 2000 & Bits \\
\hline Distance & Euclidean distance & $\sqrt{\left(x_{a}-x_{b}\right)^{2}+\left(y_{a}-y_{b}\right.}$ & Meter \\
\hline T & Node temperature & ] $35,40]$ & Degree Celsius \\
\hline
\end{tabular}


We assume $140 \mathrm{~cm} \times 180 \mathrm{~cm}$ network area composed of the sink and 11 sensor nodes (Table 1 shows the biomedical sensors used and their meanings). Figure 5 illustrates the fixed position of these biomedical sensors on the body and the links between them. It is assumed that all nodes are homogeneous concerning temperature and energy reading.

For the simulation of this model under Matlab, we defined the nodes as data structures with parameters:

- The coordinates $\mathrm{x}$ and $\mathrm{y}$ : which define their location on the body in relation to its size.

- The temperature T: the temperature of the node.

- The energy E: the residual energy of the node.

- Neighbors: the list of neighbors with their respective information: Hop count to sink, residual energy level, temperature and is it a hotspot or not.

Table 2 summarizes the different simulation parameters that we used for the simulation of the proposed protocol HTTRP.

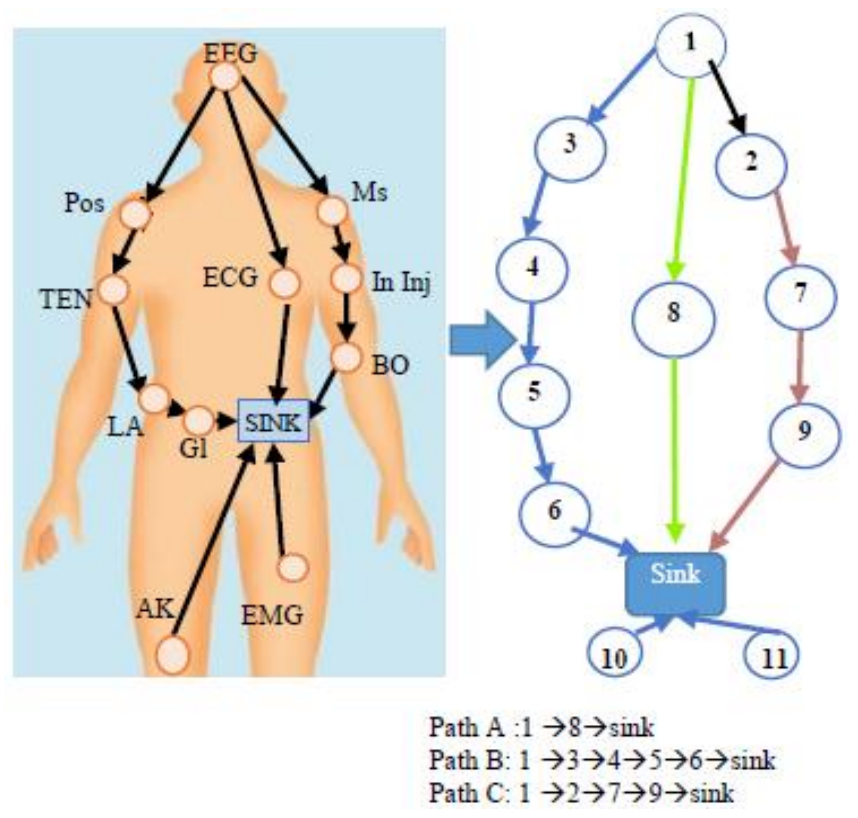

Figure 5. Simulation model

\section{SIMULATION RESULTS}

\subsection{Network lifetime}

Network lifetime is an important factor that must be considered when analyzing the performance of any algorithm for WBANs and sensor networks in general. Figure 6 shows a comparison of network lifetime between our protocol HTTRP and the TARA [15] protocol.

As we can see in the graph, the lifetime of our protocol is highly better than that of TARA (almost double). The residual energy of TARA deteriorates until the depletion of the first sensor after 2479 rounds while in our HTTRP protocol, the exhaustion of the first sensor arrives after 4836 rounds. this can be explained because TARA always selects the shortest path without taking into account the residual energy of the nodes, moreover, the withdrawal strategy adopted by TARA to remedy on hotspot detection case consumes uselessly more energy.

However, the long lifetime of HTTRP in the simulation is due to the reason that HTTRP uses node's energy efficiently.
First, it always selects the forwarder with max residual energy (i.e. the least previously solicited-node). Second, and unlike TARA, in case of hotspot detection, the hotspot node does not need to return the packet to the previous node nor send an acknowledgment message, so it saves its residual energy. Third, HTTRP uses the acknowledgment message efficiently to exchange node's temperature and residual energy so it does not need extras messages to exchange this information.

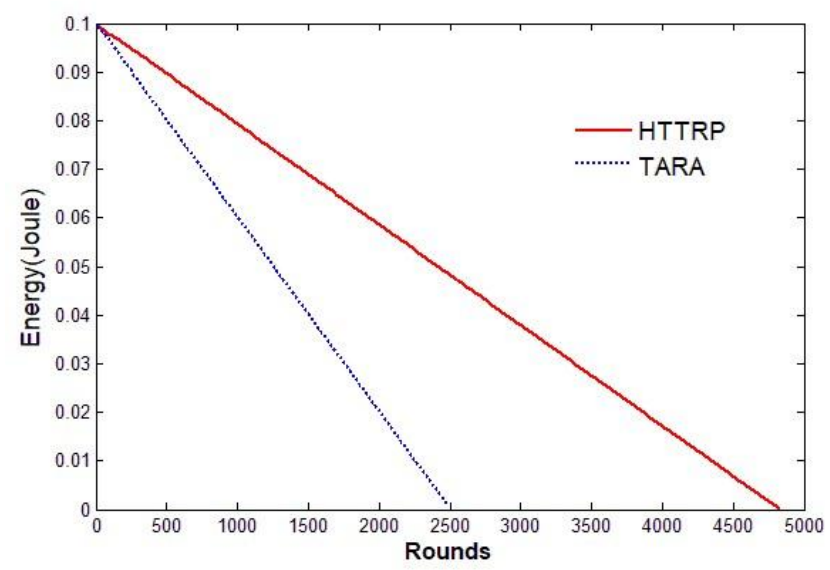

Figure 6. Network lifetime for TARA vs HTTRP

\subsection{Charge balancing}

One of the major shortcomings of TARA (and all TARP algorithms based on SHR) is the load balancing among different network nodes; hence, in this class of routing protocol, nodes in the shortest paths are highly solicited causing an unbalanced load. It can be seen in Figure 7, that TARA uses only two paths (A and B) at a very different percentage and it did not use at all the third path $\mathrm{C}$. In the simulation, TARA starts by using only the shortest path (path A) for the first 50 rounds, when it becomes unavailable (generation of the first hotspot), it starts using the second shortest path (path B), it reselects path A as soon as it becomes available and so on (Figure 8 (a)). As a result, TARA causes an unbalanced load (energy consumption and temperature rise) among the network nodes. This problem is addressed in our protocol by diversifying the routing path while dispensing uniformly the load (energy consumption and temperature rise). HTTRP uses all available paths with very close percentages (Figure 7). The charge balancing for HTTRP in the simulation is due to the reason that HTTRP always selects the colder forwarder with max residual energy, so it uses the three paths alternately (Figure 8 (b)).

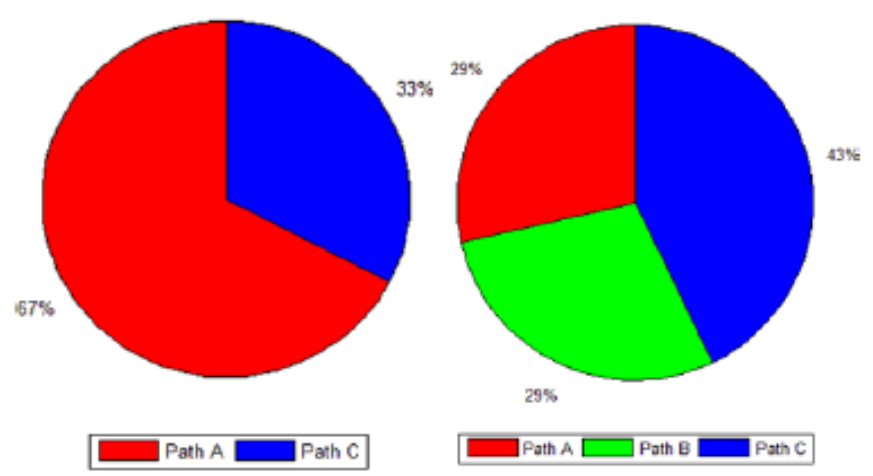

Figure 7. Percentage of the routed packet by different paths; TARA in left vs HTTRP in right 


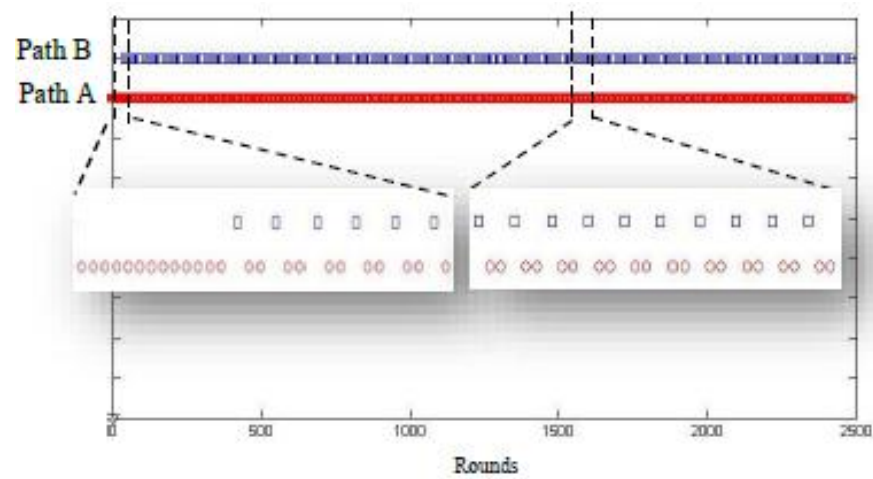

(a)

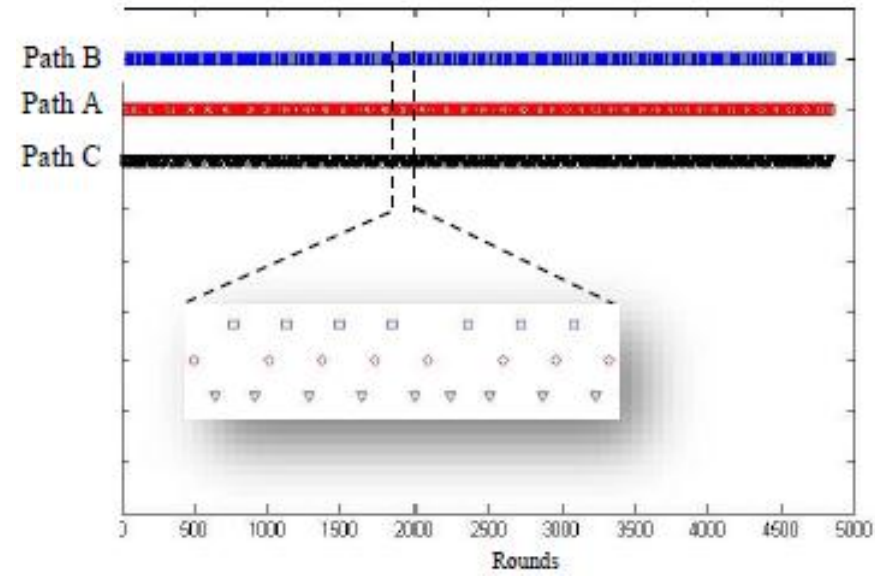

(b)

Figure 8. Path diversifying in TARA (a) and in HTTRP (b)

\subsection{Throughput}

Figure 9 represents the throughput (delivered packets in the network lifetime) comparison of HTTRP and TARA [15].

It can be observed that the overall number of delivered packets (1167 packets) HTTRP is highly bigger than that of TARA (745); this can be explained, as the network lifetime of HTTRP is longer than TARA's lifetime. However, the number of delivered packets by TARA in its lifetime (2479 rounds) is 745 packets that are bigger than 598 packets delivered by HTTRP in the same period and this because TARA uses the shortest path but our algorithm diversify the routing paths, so the packets are delivered through longer paths and therefore fewer packets are delivered in the same period.

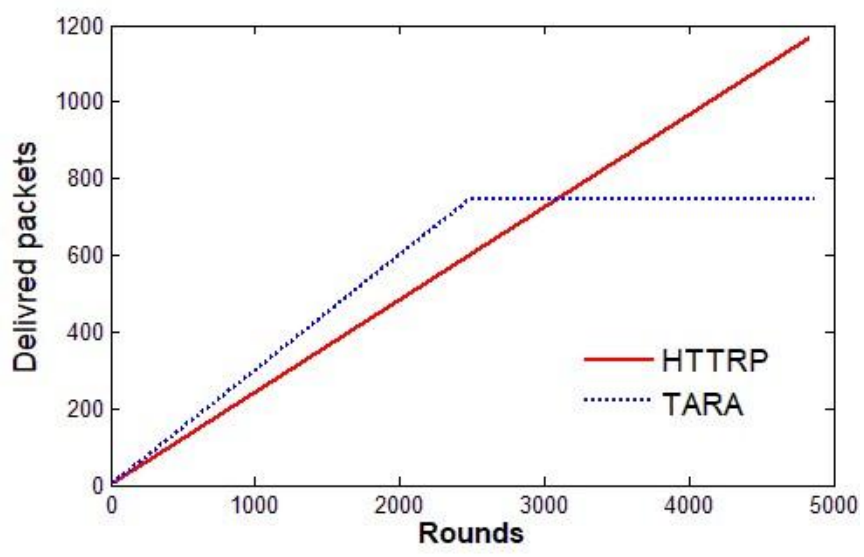

Figure 9. Throughput of TARA vs HTTRP

\subsection{Number of hotspots}

Figure 10 represents the comparison of hotspots generated during the network lifetime for both protocols. The immediate observation from this figure is the huge difference between our protocol HTTRP and the TARA protocol. TARA generates 244 hotspots in his lifetime; however, HTTRP does not generate any hotspot during its lifetime which is almost twice TARA's lifetime. This can be explained as TARA chooses always the same path until it becomes hotspot, then it selects another path. As soon as the temperature of the hotspot node drops below the threshold, it reuses it immediately, which does not allow the node to cool well, and it becomes a hotspot again very quickly. The zero hotspot for HTTRP in the simulation is due to the reason that HTTRP constantly changes the routing path which gives the nodes sufficient time to cool down.

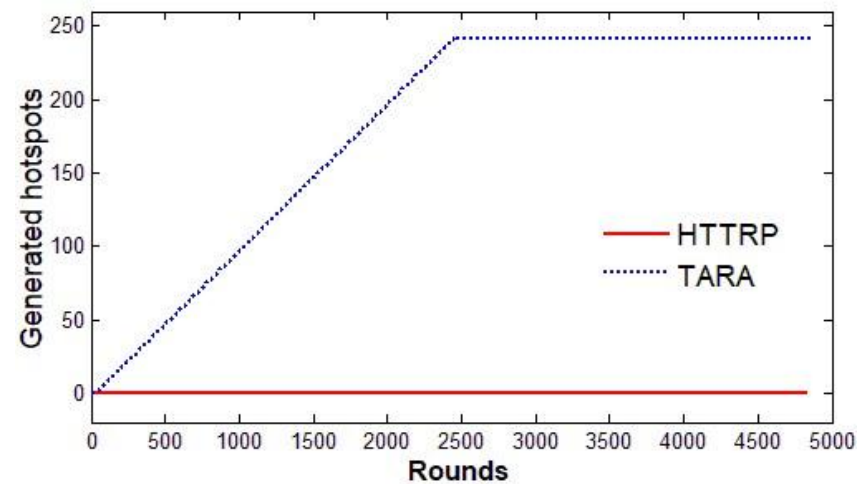

Figure 10. Hotspots generated during network lifetime (HTTRP generates 0 hotspots)

\section{CONCLUSION}

The proposals made for rooting in TARP have been numerous but they did not consider the energy of sensors during the selection of the routing paths, this has caused a problem regarding the lifetime of the network.

In this paper, we have presented HTTRP, a routing protocol for WBANs that is a thermal and energy-aware routing protocol. HTTRP takes into account the temperature and the residual energy of sensor nodes when selecting the next relay node and this in order to reduce sensors' overheating and balancing the consumption of their residual energy.

The simulations' results comparison of HTTRP with TARA protocol shown that our protocol performs very well; it prolongs the lifetime of the network by balancing the sensors' energy consumption and diversifying the routing paths. Moreover it produces a high throughput compared to TARA, in addition, it reduces overheating and avoids the creation of hotspot. These improvements are due to the selection mechanism, which is based on the residual energy and sensor's temperature. This mechanism ensures the selection of the optimal path to the sink node by equitable participation of the network's nodes.

\section{REFERENCES}

[1] Latré, B., Braem, B., Moerman, I., Blondia, C., Demeester, P. (2011). A survey on wireless body area 
networks. Wireless Networks, 17(1): 1-18 http://dx.doi.org/10.1007/s11276-010-0252-4

[2] Qu, Y., Zheng, G., Ma, H., Wang, X., Ji, B., Wu, H. (2019). A survey of routing protocols in WBAN for healthcare applications. Sensors, 19(7): 1638. http://dx.doi.org/10.3390/s19071638

[3] Kurunathan, J.H. (2015). Study and overview on WBAN under IEEE 802.15. 6. U. Porto Journal of Engineering, 1(1): $\quad 11-21$. 6493_001.001_0003

[4] Zuhra, F.T., Bakar, K.A., Ahmed, A., Tunio, M.A. (2017). Routing protocols in wireless body sensor networks: A comprehensive survey. Journal of Network and Computer Applications, 99: 73-97. http://dx.doi.org/10.1016/j.jnca.2017.10.002

[5] Hasan, K., Biswas, K., Ahmed, K., Nafi, N.S., Islam, M.S. (2019). A comprehensive review of wireless body area network. Journal of Network and Computer Applications, 143: 178-198. http://dx.doi.org/10.1016/j.jnca.2019.06.016

[6] Movassaghi, S., Abolhasan, M., Lipman, J., Smith, D., Jamalipour, A. (2014). Wireless body area networks: A survey. IEEE Communications Surveys \& Tutorials, 16(3):

$1658-1686$ http://dx.doi.org/10.1109/SURV.2013.121313.00064

[7] Baig, M.M. (2014). Smart vital signs monitoring and novel falls prediction system for older adults. Auckland University of Technology.

[8] Olatinwo, D.D., Abu-Mahfouz, A., Hancke, G.J.S. (2019). A survey on LPWAN technologies in WBAN for remote health-care monitoring. Sensors, 19(23): 5268. http://dx.doi.org/10.3390/s19235268

[9] Monowar, M.M., Mehedi Hassan, M., Bajaber, F., Hamid, M.A., Alamri, A. (2014). Thermal-aware multiconstrained intrabody QoS routing for wireless body area networks. International Journal of Distributed Sensor $\quad$ Networks, $10(3)$ : 676312. http://dx.doi.org/10.1007/s11276-010-0252-4

[10] Shehu, N.M., Adam, M.M. (2016). A survey on thermal aware routing protocols in WBAN. Science [ETEBMS2016], 5: 6 .

[11] Ahmed, G., Islam, S.U., Shahid, M., Akhunzada, A., Jabbar, S., Khan, M.K., Riaz, M., Han, K.J. (2018). Rigorous analysis and evaluation of specific absorption rate (SAR) for mobile multimedia healthcare. IEEE Access, 6: 29602-29610. http://dx.doi.org/10.1109/ACCESS.2018.2839909

[12] Ntouni, G.D., Lioumpas, A.S., Nikita, K.S. (2014). Reliable and energy-efficient communications for wireless biomedical implant systems. IEEE Journal of Biomedical and Health Informatics, 18(6): 1848-1856. http://dx.doi.org/10.1109/JBHI.2014.2300151

[13] Jiang, W., Wang, Z., Feng, M., Miao, T. (2017). A survey of thermal-aware routing protocols in wireless body area networks. In 2017 IEEE International Conference on Computational Science and Engineering (CSE) and IEEE International Conference on Embedded and Ubiquitous Computing (EUC), Guangzhou, China. http://dx.doi.org/10.1109/CSE-EUC.2017.189

[14] Oey, C.H.W., Moh, S. (2013). A survey on temperatureaware routing protocols in wireless body sensor networks. Sensors, $\quad$ 13(8): $\quad 9860-9877$. http://dx.doi.org/10.3390/s130809860
[15] Tang, Q., Tummala, N., Gupta, S.K., Schwiebert, L. (2005). TARA: Thermal-aware routing algorithm for implanted sensor networks. In: Prasanna, V.K., Iyengar, S.S., Spirakis, P.G., Welsh, M. (eds), Distributed Computing in Sensor Systems. DCOSS 2005. Lecture Notes in Computer Science, vol 3560. Springer, Berlin, Heidelberg. http://dx.doi.org/10.1007/11502593_17

[16] Bag, A., Bassiouni, M.A. (2006). Energy efficient thermal aware routing algorithms for embedded biomedical sensor networks. In 2006 IEEE International Conference on Mobile Ad Hoc and Sensor Systems, Vancouver, BC, Canada. http://dx.doi.org/10.1109/MOBHOC.2006.278619

[17] Takahashi, D., Xiao, Y., Hu, F., Chen, J., Sun, Y. (2008). Temperature-aware routing for telemedicine applications in embedded biomedical sensor networks. EURASIP Journal on Wireless Communications and Networking, 572636. http://dx.doi.org/10.1155/2008/572636

[18] Bag, A., Bassiouni, M.A. (2008). Hotspot preventing routing algorithm for delay-sensitive applications of in vivo biomedical sensor networks. Information Fusion, $9(3)$ : 389-398. http://dx.doi.org/10.1016/j.inffus.2007.02.001

[19] Bag, A., Bassiouni, M.A. (2008). Routing algorithm for network of homogeneous and id-less biomedical sensor nodes (RAIN). In 2008 IEEE Sensors Applications Symposium, Atlanta, GA, USA, pp. 68-73. http://dx.doi.org/10.1109/SAS13374.2008.4472946

[20] Ahourai, F., Tabandeh, M., Jahed, M., Moradi, S. (2009). A thermal-aware shortest hop routing algorithm for in vivo biomedical sensor networks. In 2009 Sixth International Conference on Information Technology: New Generations, Las Vegas, NV, USA, pp. 1612-1613. http://dx.doi.org/10.1109/ITNG.2009.274

[21] Rafatkhah, O., Lighvan, M.Z. (2014). M2E2: A novel multi-hop routing protocol for wireless body sensor networks. International Journal of Computer Networks and Communications Security (IJCNCS), 2(8): 260-267.

[22] Bhangwar, A.R., Kumar, P., Ahmed, A., Channa, M.I. (2017). Trust and thermal aware routing protocol (TTRP) for wireless body area networks. Wireless Personal Communications, $\quad$ 97(1): 349-364. http://dx.doi.org/10.1007/s11277-017-4508-5

[23] Jamil, F., Iqbal, M.A., Amin, R., Kim, D. (2019). Adaptive thermal-aware routing protocol for wireless body area network. Electronics, 8(1): 47. http://dx.doi.org/10.3390/electronics8010047

[24] Heinzelman, W.R., Chandrakasan, A., Balakrishnan, H. (2000). Energy-efficient communication protocol for wireless microsensor networks. In Proceedings of the 33rd annual Hawaii International Conference on System Sciences, Maui, HI, USA, p. 10. http://dx.doi.org/10.1109/HICSS.2000.926982

[25] Ullah, S., Higgins, H., Braem, B., Latre, B., Blondia, C., Moerman, I., Saleem, S., Rahman, Z., Kwak, K.S. (2012). A comprehensive survey of wireless body area networks. Journal of Medical Systems, 36(3): 1065-1094. http://dx.doi.org/10.1007/s10916-010-9571-3

[26] Ahmed, G., Mahmood, D., Islam, S. (2019). Thermal and energy aware routing in wireless body area networks. International Journal of Distributed Sensor Networks, 15(6). https://doi.org/10.1177/1550147719854974 